\title{
ERRATUM TO: Analysis of two heterogeneous server queueing model with balking, reneging and feedback Mathematical Sciences And Applications E-Notes, 2, No. 2, (2014), 10-21
}

\author{
Amina Angelika BOUCHENTOUF*, Mokhtar KADI and Abbes RABHI
}

(Communicated by İ Onur KIYMAZ)

1. On page 04 , after equation (3.2), we should have

$$
\left(\lambda+\beta\left(\mu_{1}+\mu_{2}\right)\right) P_{2}=\left(\beta\left(\mu_{1}+\mu_{2}\right)+\eta \delta\right) P_{3}+\lambda P_{1}, \quad n=2 .
$$

2. Instead of equation (3.3), we should have

$$
\left(\frac{\lambda}{n-1}+\beta\left(\mu_{1}+\mu_{2}\right)+(n-2) \eta \delta\right) P_{n}=\left(\beta\left(\mu_{1}+\mu_{2}\right)+(n-1) \eta \delta\right) P_{n+1}+\frac{\lambda}{n-2} P_{n-1}, \quad 3 \leq n \leq N-1
$$

\section{Affiliations}

AMINA ANGELIKA BOUCHENTOUF

AdDREss: Department of Mathematics, Djillali Liabes University of Sidi Bel Abbes, B. P. 89, Sidi Bel Abbes 22000, Algeria.

E-MAIL: bouchentouf_amina@yahoo.fr

MOKHTAR KADI

AdDRESS: Department of Mathematics and Computer Sciences, Moulay Tahar University, Saida, B.P. 138, En-Nasr Saida 20 000, Algeria.

E-MAIL: kadi1969@yahoo.fr

ABBES RABHI

AdDress: Department of Mathematics, Djillali Liabes University of Sidi Bel Abbes, B. P. 89, Sidi Bel Abbes 22000, Algeria.

E-MAIL: rabhi_abbes@yahoo.fr 